\title{
Age Difference in Recognition of Emoticons
}

\author{
Kun-An Hsiao and Pei-Ling Hsieh \\ Industrial Design Department, \\ National Kaohsiung Normal University \\ No.62, Shenjhong Rd., Yanchao, Kaohsiung, Taiwan \\ kahsiao@nknucc.nknu.edu.tw
}

\begin{abstract}
Emoticons are new visual or nonverbal communication cues used in digital interaction. Can elders recognize and discriminate emotional states from emoticons as well as young people can? This study uses emoticons to investigate recognizable emotional differences between thirty older and thirty younger adults. A total of 32 representative emoticons were used as stimuli, consisting of 24 realistic facial expressions, 8 abstract facial expressions, 11 positive emotions, 11 neutral emotions, and 10 negative emotions. Four questions, including "emotional state," "imitative level," "preference," and "understanding" of emoticons, were applied in the questionnaire. The results showed older adults to have a more positive response toward perceived emoticons than younger adults. The two age ranges have different cognition of the design appearances of realistic and abstract emoticons. Older adults showed less preference toward all emoticons than younger adults. The result also denotes older adults can analogize between realistic faces and emoticons.
\end{abstract}

Keywords: Age difference, Emoticon, Recognition.

\section{Introduction}

"Emoticon" is a portmanteau word formed from "emotion" and "icon." Initially, it was a textual face composed of punctuation-marks to convey one's mood or facial expression, first used on the Internet in 1982. Emoticons are commonly applied in email messages, instant messaging or short messages as graphic representations of facial expressions. Walther and D'Addario [1] described that emoticons outweighed verbal content. Derks, Bos and Grumbkow [2] also indicated that using emoticons could inspire people's social meaning from messages. Huang, Yen and Zhang [3] pointed out that emoticons were not only enjoyable to use but also a helpful communication method. Their results showed that users could experience enjoyment, adding richness and utility to information, through emoticons.

In previous studies, handling different types of emotional information, elders presented a broader advantage in detecting these stimuli [4], and tended to experience or remember more positive emotions than young adults [5][6][7][8]. Older adults also easily memorized emotional goods, even slogans [9]. Appropriate strategies to manage decline of cognitive ability or neural deficits from aging become important for 
most elders. Mather and Carstensen [10] indicated that, compared with younger adults, strategic processes play a greater role in older adults' emotional attention and memory. Older adults showed more emotionally pleasant memory distortion for past choices and autobiographical information.

Socioemotional selectivity theory (SST) employs subjective studies of human emotion and behavior to propose an impressive and effective perspective interpreting cognition and changing behavior of older adults. Carstensen, Isaacowitz, \& Charles [11] demonstrated the socio-emotional goal setting of individuals depends on perceived time left in life. One significant psychological goal in SST is the entire goal (like obtaining knowledge or a new social relationship); another is correlated with emotion (such as balancing emotions or feeling needed by others) [12]. Older adults realized that when future life is limited, they should prioritize social motives in an optimal goal setting to acquire more satisfying experience from social relationships and make more positive choices. People regulate their emotional states to optimize psychological well-being [13]. These studies demonstrated that elders tend to appear happy with a positive mental attitude in daily life. Would these emotional behaviors and responses of elders be similar when relating to natural or artificial objects?

Lee, Tang, Yu and Cheung [14] indicated that the emoticon was a simple, nonlanguage-based, culturally neutral, non-verbal and easily-applied tool for investigation. They applied simple emoticons to assess patients' mood status and the results were comparable to Geriatric Depression Scale (GDS). For comparison with prior results, this study employs emoticons as experimental stimuli to explore differences of emotional cognition between older and young adults.

However, given the new visual cues of digital interaction, can elders recognize emoticons as well as young people do? Can they discriminate states of emotion from emoticons that convey facial expression and new social meanings? This study examines the following hypotheses: (H1) older adults can distinguish the emotion differences of emoticons such as normal emotional stimuli, $(\mathrm{H} 2)$ realistic emoticons are more easily recognized than abstract emoticons and (H3) older adults will experience greater positive emotions toward emoticons than younger adults.

\section{$2 \quad$ Methodology}

\subsection{Participants}

Thirty younger adults ( 15 females, 15 males, mean age $=22.5$ years, age range: $19-26$ years) were recruited from the Chang Gung University. Thirty older adults (18 females, 12 males, mean age $=79.4$ years, age range: $67-91$ years) were recruited through two Senior Citizen Centers of Taiwan. They received a gift for their participation after the experiment. All participants had normal or corrected-to-normal visual acuity and no visual pathologies. There were eight additional participants: four younger adults (mean age $=26.5$ years) and four older adults (mean age $=78$ years), recruited in the same way as above to provide pilot test results for this study. 


\subsection{Material}

This study applied familiar emoticons, commonly seen on MSN and interfaces of digital products collected from the Internet, as stimuli. Although emoticons range from facial or bodily expression in real photos to abstract punctuation-marks in the MSN application, this study emphasized design icons with facial expressions. Firstly, two hundred and fifty-two emoticons were widely collected from an iconic design website for instant messaging (IM) and text messaging (TM) software, representing facial expression or mood. After eliminating similar and blurred emoticons, a focus group comprising five designers (mean age $=27$ years) with more than five years' design discipline and experience with emoticons were assigned to judge and categorize representative emoticons for further investigation. The features of representative emoticons will be taken as bases in related analyses of this study. The mode of presentation indicates whether emoticons are graphic icons or punctuation-mark icons. Therefore, the focus group needed to classify representative emoticons according to emotional expression and realistic design appearance.

The focus group was first asked to choose emoticons not only including the same design style, but also displaying different facial expressions. It then arranged the emoticons into "three emotional states:" positive emotion, neutral emotion and negative emotion. The next step was to sort emoticons into "two imitative levels," realistic and abstract facial expression, in each emoticon style. The experimental stimuli were controlled considering the elders' physiological and psychological capacity in judging emotional tests. Finally, the focus group extracted four emoticon styles including thirty-two emoticons as representative stimuli and basic categories in this study. Figure 1 shows the thirty-two emoticons consisting of twenty-four realistic facial expressions, eight abstract facial expressions, eleven positive emotions, eleven neutral emotions and ten negative emotions. There were four emoticon styles containing illustrated icons (Style a. from No. 1 to No. 6), three-dimensional facial icons (style b. from No. 7 to No. 15), two-dimensional facial icons (Style c. from No. 16 to No. 24) and punctuation-mark representation (Style d. from No. 25 to No. 32). To avoid color effects, including hue, value, chroma, and the interaction between colors and shapes, the experiment used grayscale emoticons.

Each sheet of the questionnaire included a first stage of personal information: age, gender, education, computer experience and emotional self-assessment on a 7-point Likert scale from sad to happy $(1=\operatorname{sad}, 4=$ neutral feeling and $7=$ happy $)$. The second stage randomly arranged each emoticon in a questionnaire booklet and each page included one representative emoticon and four questions: "emotional state", "imitative level," "preference," and "understanding." The judgment of "emotional state" evaluated the emotional expression of emoticons on a 7-point scale from negative to positive ( $1=$ extremely negative, $4=$ neutral and $7=$ extremely positive $)$. The judgment of "imitative levels" evaluated the degree of emotional realism on a 7-point scale from 1 (extremely abstract) to 7 (extremely realistic), to verify Hypothesis 2 . The judgment of "preference" evaluated the degree of favor toward emoticons on a 7point scale $(1=$ dislike very much, and $7=$ like very much $)$, allowing evaluation of the difference in preference toward different emoticons between two age subjects. 
The additional question "understanding of this emoticon" clarified whether subjects realized what each emoticon represented. Subjects answered intuitively whether they could understand the emoticon $(1=$ yes, $2=$ no $)$.

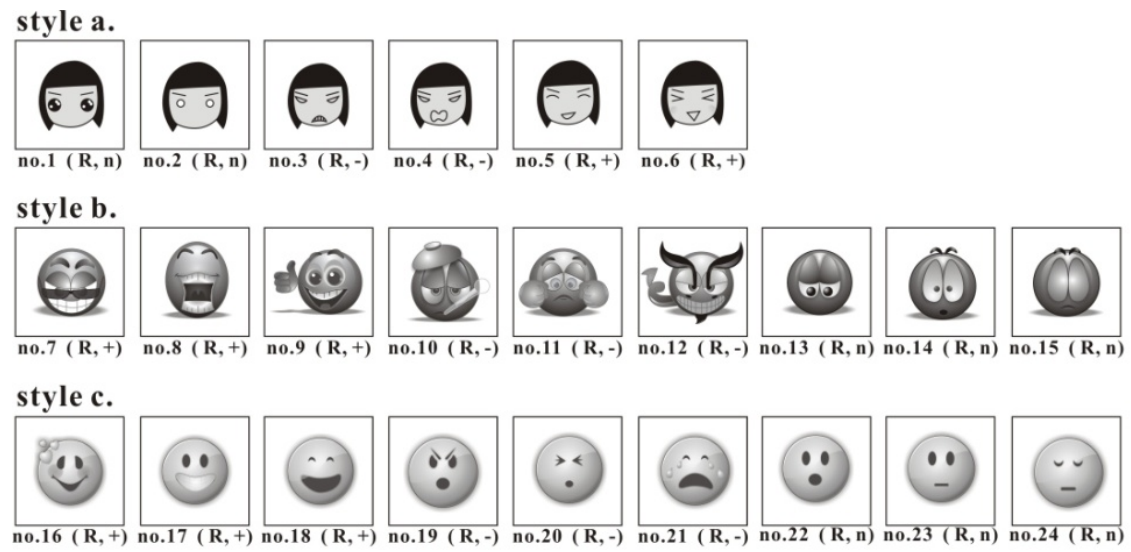

style d.

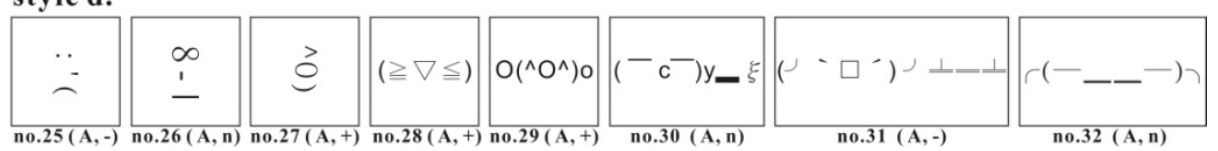

(A: Abstract, R: Realistic; +: positive emotion, -: negative emotion, n: neutral emotion)

Fig. 1. 32 representative emoticons

\subsection{Procedures}

Before answering the questionnaire, subjects had an introduction to the experiment's purpose and procedure, and then filled in personal data. Additionally, all subjects also needed to describe an example of an icon and to read a question aloud on the first test page to confirm their normal vision. The researchers accompanied each subject in whole process.

\section{$3 \quad$ Results}

\subsection{Self-assessment of Emotion}

Comparing the responses of emotional self-assessment between older and younger adults, this study applied an independent $T$ test to analyze the judgments of the two age groups. The result showed a significant difference between older adults $(\mathrm{M}=5.77$; S.D. =1.55) and younger adults $(\mathrm{M}=4.73$; S.D. $=1.17)(\mathrm{F}=3.664 ; \mathrm{P}=0.005<0.01)$ : older adults reported more pleasure than younger adults under normal conditions. 


\subsection{Understanding of Emoticons}

Older adults understood $70 \%$ of all emoticons, compared to $83 \%$ with younger adults. The understanding of abstract emoticons was obviously lower (from No. 25 to No. 32, the average understanding rate is $47 \%$ ) than realistic emoticons (from No. 1 to No. 24, average understanding rate is $88 \%$ ). Comprehending abstract emoticons was difficult for both subject groups (30\% of older adults, compared to $63 \%$ of younger adults). However, eliminating the abstract emoticons, the understanding rate increases to $80 \%$ with older adults and $93 \%$ among younger adults. Textual emoticons, therefore, are more difficult to understand than graphic emoticons, particularly for elders. In particular, the rate of understanding for Nos. 27, 30 and 31 is lower than $20 \%$ with older adults; for no. 26 and no. 27 it is lower than $30 \%$ with younger adults. Researchers found that these emoticons are either unlike facial images, or too complex, using so many punctuation marks that subjects can hardly recognize their contents. Comparing the comprehension rate among four emoticon styles, Style c. (90\%) was higher than Style a. (86\%), Style b. (73\%) and Style d. (30\%) for older adults, and Style c. (96\%) was also higher than Style a. (93\%), Style b. (90\%) and Style d. (63\%) for younger adults. Style c. presented simple two-dimensional facial icons, and delivered easily-understood emotional expression. Emoticons of Style d. expressed abstract combinations with punctuation-marks, delivering unfamiliar images that were difficult to understand.

\subsection{Emotional States}

Firstly, researchers tested the correlation between categories by focus group and subjects' judgments of thirty-two emoticons expressing three emotional states. The results show positive correlation for both older adults $(0.783, \mathrm{P}<0.01)$ and younger adults $(0.902, \mathrm{P}<0.01)$, meaning the discrimination of emotional states was similar between the focus group and two subject groups. To examine the judgments of emotional state on all emoticons, this study applied an independent $\mathrm{T}$ test to analyze the judgments of two age groups. Older adults and younger adults differed significantly from each other $(\mathrm{F}=6.115 ; \mathrm{P}=0.002<0.01)$. On average, the older adults $(\mathrm{M}=4.24$; S.D. =1.87) feel more positive emotion for emoticons than younger adults $(M=3.98$; S.D. =1.92), whether they understand them or not. Removing abstract emoticons, the effect of emotional state on judgment of realistic emoticons is also significantly different between two different age groups $(\mathrm{F}=1.619 ; \mathrm{P}=0.001<0.01)$. Older adults rated emoticons more than $4(\mathrm{M}=4.29$; S.D. $=2.05)$ more often than younger adults $(\mathrm{M}=3.95$; S.D. $=1.98)$, revealing a more positive reaction to emoticons. Figure 2 shows the dissimilar proportion of average scores in three emotional states between the two age groups. Further exploring average scores of three emotional states by two subject groups, the ANOVA test showed significant differences among positive, neutral and negative emotion states by older adults $(\mathrm{F}(2,957)=81.996 ; \mathrm{P}=0.000<0.01)$ and younger adults $(\mathrm{F}(2,957)=646.658 ; \mathrm{P}=0.000<0.01)$. The judgments of younger adults ranged broadly from 2.30 to 5.84 points, but those of older adults centered between 3.08 and 5.14 points. Older adults used more ambiguous and positive emotional criteria responding to these emoticons. These results verify H3: older adults will respond with greater positive emotion to emoticons than younger adults. 


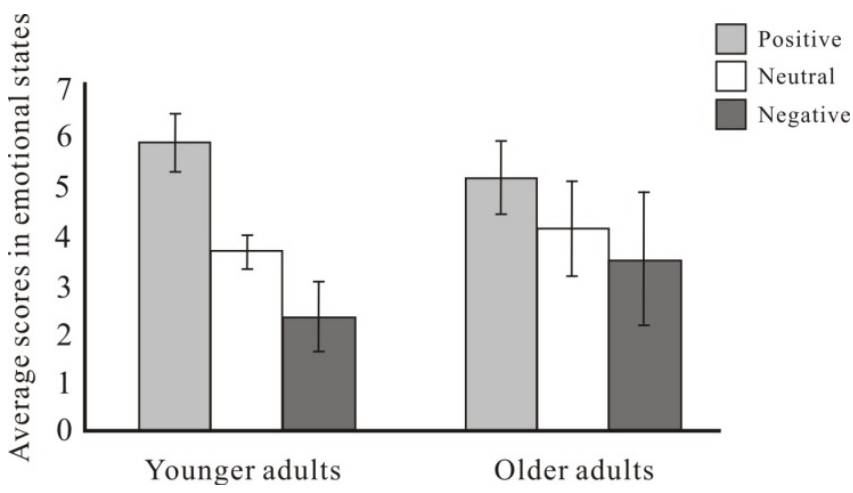

Fig. 2. Average scores of three emotional states by two age groups

\subsection{Imitative Levels}

On judgments of two imitative levels, older and younger adults differed significantly $(\mathrm{F}=7.572 ; \mathrm{P}=0.019<0.05)$ on all emoticons, also differing significantly on realistic emoticons $\quad(\mathrm{F}=111.144 ; \quad \mathrm{P}=0.000<0.01) \quad$ and abstract emoticons $\quad(\mathrm{F}=7.234$; $\mathrm{P}=0.001<0.01)$ separately. The results show that the two age groups have different recognition of design appearances for realistic and abstract emoticons. For older adults, emoticons Nos. 10,13 and 15 (realistic design) were evaluated as abstract $(M=3.86<4)$, while no emoticon belonging to abstract design was evaluated as realistic $(M \leqq 4)$. For younger adults, only emoticon No. $15(M=3.90)$ (realistic design) was evaluated as abstract; and No. 28 ( $\mathrm{M}=4.30)$, No. $29(\mathrm{M}=4.33)$ emoticons (abstract design), were evaluated as realistic. Both groups judged most emoticons composed of punctuation marks lower than 4 , meaning both age groups considered this type of emoticon an abstract design. The responses of younger adults (from 1.50, highly abstract, to 6.23, highly realistic) appeared more extreme than older adults (from 3.13 to 6.17). The results demonstrate that $\mathrm{H} 2$, realistic emoticons are easier to understand than abstract emoticons, is tenable.

\subsection{Preference}

There were significant differences in preference judgments between older adults and younger adults $(\mathrm{F}=98.512 ; \mathrm{P}=0.000<0.01)$ for the thirty-two emoticons. The average preference scores of older adults $(\mathrm{M}=3.79$; S.D. $=2.06)$ were lower than those of younger adults $(M=4.21$; S.D. $=1.58)$, indicating that older adults had less preference toward all emoticons than did younger adults. Taking the two imitative levels separately, older adults and younger adults differed significantly on realistic emoticons $(\mathrm{F}=173.521 ; \mathrm{P}=0.002<0.01)$ and abstract emoticons $(\mathrm{F}=1.787 ; \mathrm{P}=0.000<0.01)$. Older adults $(\mathrm{M}=3.92$; S.D. $=2.18)$ gave lower preference scores than younger adults $(\mathrm{M}=4.22$; S.D. $=1.54)$ for both realistic and abstract emoticons, the same scores from older adults $(M=3.43$; S.D. $=1.58)$ were also lower than younger adults $(M=4.17$; S.D. $=1.70$ ). Younger adults mainly gave scores greater than 4 on both imitative levels, 
meaning they tend to like those emoticons. By contrast, older adults usually scored them less than 4 , meaning they tend to dislike those emoticons, especially abstract emoticons composed of punctuation-marks.

\section{Conclusion}

This study intended to interpret recognizable differences toward emoticons between younger and older adults by experimental design and analysis. Examining test data in this study and referencing previous studies reveals the diversity between different age groups and provides appropriate information of emoticon design for elderly people in the future digital era.

\subsection{Design Effects of Emoticons}

Based on the comprehension rate for all emoticons, researchers found that design effects strongly affected recognition of the emoticon. This included two aspects. First were the important features extracted from facial expression, and second was the quantity of information. Comparing facial organs or features among emoticon styles a., b. and c., Style c., mainly depicting only eyes and mouth, got the highest understanding rate. Emoticons such as Nos. 1, 2, 13 and 15, without or having unclear mouth features, elicit a lower understanding rate. This implies that eyes and mouth were key facial features delivering most emotional information. Other organs like eyebrows, teeth or nose, etc. had lower effect on emotional delivery. Regarding quantity of information, emoticons designed with too many unrelated features like hair, shadows or exaggerated expressions on eyes or mouth interfered with emotional recognition and caused the opposite effect. Although Style d. (using punctuation marks) generated the lowest understanding, especially for elder subjects ( $M=30 \%$; S.D. $=13 \%$ ), No. 25 still acquired 57\% understanding for elder subjects. Emoticon 25 applied a colon as eyes, and a bracket to simulate an unhappy mouth. This emoticon made of punctuation marks appropriately manipulated two design aspects portraying important facial features and a clear image to create a recognizable emotion. It proved designs must correlate with user's knowledge of the world [15].

\subsection{Emotional Recognition of Elders}

The results of emotional self-assessment and emoticon recognition of three emotional states showed significant differences between older and younger adults, where older adults feel more pleasure and positive emotion than younger adults. Older adults displayed more positive responses than younger adults to perceived artificial designs of emoticons. This confirmed similar results from past studies of emotional cognition when judging living objects such as human faces or natural objects $[4][16][10][17]$. Therefore, researchers inferred that there was same judging valence of emotional cognition between living and non-living objects by elders. It also denotes older adults can analogize between realistic faces and varied emoticons by designs merging differ- 
ent emotional expressions. These results verify the tenability of $\mathrm{H} 1$, that older adults could distinguish the emotional differences of emoticons equally well as normal emotional stimuli. The results also could give evidences for further emoticon application and related designs on product interfaces or information communication for elders.

Why did elders feel more positive towards these emoticons? Socioemotional selectivity theory proposes that elders tend to shift towards emotionally meaningful goals and to satisfy or stabilize their emotional needs in social networks and objects in a limited time left to live [5]. Elders would therefore pay more attention to an important emotional target. Especially in attention and memory, elders would prefer positive and prevent negative information [10]. The activated brain states of elders also differ from young adults in processing and responding to different emotional stimuli [18] [19][20]. The emoticons represented the familiar human face and contained plentiful emotional expression, echoing arguments above. These could explain the cognitive differences of emoticons between two subject groups.

Judgment of emoticons' cognition differences had a broad range for younger adults but centralized for elder adults. Older adults kept more positive but ambiguous emotional criterion to response these emoticons. Charles, Mather and Carstensen [21] examined age differences in recall and recognition memory for emotional images also found younger adults show a wider portion of negative images than positive and neutral objects, but older and middle-age adults showed parallel emotional valence. Schaie [22] pointed out the cognitive ability of elders stayed high until around age 60, and then began to decline. The gradual decline of body and mind pushes elders to control moods and avoid extreme effects on both physiology and psychology. This caused older adults' recognition of emoticons to be more positive and centralized than younger adults.

\subsection{Emoticon Preference of Elders}

The preference scores of elders were smaller than the neutral score of 4 , meaning they did not prefer many emoticons, especially those composed of punctuation marks. Young people commonly use these in online communicating tools such as bulletin boards, online chat, instant messaging and e-mail interfaces [23]. In this study, only two elders had experience with computers, meaning elders almost never used the emoticons. That is why older subjects showed lower understanding and unfamiliarity toward those emoticons. Why were the emotional judgments of elders toward emoticons higher and more positive than young adults, but their preference judgments significantly lower than those of younger subjects? For complex emotional cognition, Ross \& Mirowsky [24] explained, elders possess both maturity and decline, which could be why older people report more positive emotions and more passive emotions simultaneously. The preferences are a process under many aspects of the decision environment and the judgment of preferences could be regarded the choice of objects [25][26]. Elders have the most common emotions like contentment, calm, and ease but of the elation and excitement [24]. These novel and unfamiliar emoticons obviously could not get the preferences by older people. 


\subsection{Limitations and Further Studies}

Most elders had little experience using these interface elements. The using experiences to be an important factor influencing emotion and preference toward these emoticons. Though older adults may favor the familiar and dislike novel things, these emoticons seem to have innately new characteristics and also affect evaluative judgment. For young adults, these emoticon designs will be a common tool in their daily life when they become elders.

Although the artificial emoticon designs were the main subject in this study, it found age differences regarding emotional states and preference. Are there also existing age and recognition differences for comparing facial expressions between real human faces and emoticon? Can real human faces elicit more positive emotion and better preference than emoticons? Are these findings useful for elders on further application of digital communication? These questions merit further investigation.

Acknowledgment. This research was supported by the National Science Council of Taiwan under grant NSC98-2410-H-182-018.

\section{References}

1. Walther, J.B., D'Addario, K.P.: The Impacts of Emoticons on Message Interpretation in Computer-Mediated Communication. Social Science Computer Review 19(3), 324-347 (2001)

2. Derks, D., Bos, A.E.R., Grumbkow, J.V.: Emoticons and Social Interaction on theInternet: the Importance of Social Context. Computers in Human Behavior 23, 842-849 (2007)

3. Huang, A.H., Yen, D.C., Zhang, X.: Exploring the Potential Effects of Emoticons. Information \& Management 45(7), 466-473 (2008)

4. Leclerc, C.M., Kensinger, E.A.: Effects of Age on Detection of EmotionalInformation. Psychology and Aging 23(1), 209-215 (2008)

5. Carstensen, L.L., Pasupathi, M., Mayr, U., Nesselroade, J.R.: Emotional Experience in Everyday Life across the Adult Life Span. Journal of Personality and Social Psychology 79(4), 644-655 (2000)

6. Charles, S.T., Reynolds, C.A., Gatz, M.: Age-Related Differences and Change in Positive and Negative Affect Over 23 Years. Personality and Social Psychology 80(1), 136-151 (2001)

7. Gross, J.J., Carstensen, L.L., Tsai, J., Skorpen, C.G., Hsu, A.Y.C.: Emotion and Aging: Experience, Expression, and Control. Psychology and Aging 12(4), 590-599 (1997)

8. Mather, M., Canli, T., English, T., Whitfield, S., Wais, P., Ochsner, K., Gabrieli, J.D.E., Carstensen, L.L.: Amygdala Responses to Emotionally Valenced Stimuli in Older and Younger Adults. Psychological Science 15(4), 259-263 (2004)

9. Carstensen, L.L., Mikels, J.A.: At the Intersection of Emotionand Cognition - Aging and the Positivity Effect. Current Directions in Psychological Science 14(3), 117-121 (2005)

10. Mather, M., Carstensen, L.L.: Aging and Motivated Cognition: the Positivity Effect in Attention and Memory. Trends in Cognitive Sciences 9(10), 496-502 (2005)

11. Carstensen, L.L., Isaacowitz, D.M., Charles, S.T.: Taking Time Seriously. A Theory of Socioemotional Selectivity. Am. Psychol. 54(3), 165-181 (1999) 
12. Carstensen, L.L., Fung, H.H., Charles, T.S.: Socioemotional Selectivity Theory and the Regulation of Emotion in the Second Half of Life. Motivation and Emotion 27(2), 103123 (2003)

13. Carstensen, L.L.: The Influence of a Sense of Time on Human Development. Science 312(5782), 1913-1915 (2006)

14. Lee, A.C.K., Tang, S.W., Yu, G.K.K., Cheung, R.T.F.: The smiley as a simple screening tool for depression after stroke: A preliminary study. International Journal of Nursing Studies 45(7), 1081-1089 (2008)

15. Norman, D.: The Design of Everyday Things. Doubleday, NewYork (1988)

16. Lee, T.M.C., Ng, E.H.H., Tang, S.W., Chan, C.C.H.: Effects of Sad Moodon Facial Emotion Recognition in Chinese People. Psychiatry Research 159, 37-43 (2008)

17. Mikels, J.A., Larkin, G.R., Reuter-Lorenz, P.A., Carstensen, L.L.: Divergent Trajectories in the Aging Mind: Changes in Working Memory for Affective Versus Visual Information with Age. Psychol Aging 20(4), 542-553 (2005)

18. Braver, T.S., Barch, D.M.: A Theory of Cognitive Control, AgingCognition, and Neuromodulation. Neuroscience and Biobehavioral Reviews 26, 809-817 (2002)

19. Gunning-Dixon, F.M., Gur, R.C., Perkins, A.C., Schroeder, L., Turner, T., Turetsky, B.I., Chan, R.M., Loughead, J.W., Alsop, D.C., Maldjian, J., Gur, R.E.: Age-related Differences in Brain Activation during EmotionalFace Processing. Neurobiology of Aging 24, 285295 (2003)

20. Hedden, T., Gabrieli, J.D.E.: Insights into the Ageing Mind: A View fromCognitive Neuroscience. Nature Reviews Neuroscience 5, 87-96 (2004)

21. Charles, S.T., Mather, M., Carstensen, L.L.: Aging and Emotional Memory: The Forgettable Nature of Negative Images for Older Adults. Journal of Experimental Psychology: Genarl 132(2), 310-324 (2003)

22. Schaie, K.W.: Intellectual Development in Adulthood: The Seattle Longitudinal Study. Cambridge Univ. Press, Cambridge (1996)

23. Mock, K.: The Use of Internet Tools to Supplement Communication in The Classroom. Journal of Computing Sciences in Colleges 17(2), 14-21 (2001)

24. Ross, C.E., Mirowsky, J.: Age and The Balance of Emotions. Social Science \& Medicine 66, 2391-2400 (2008)

25. Sharot, T., De Martino, B., Dolan, R.J.: How Choice Reveals and Shapes Expected Hedonic Outcome. Journal of Neuroscience 29, 3760-3765 (2009)

26. Lichtenstein, S., Slovic, P. (eds.): Construction of Preferences. Cambridge University Press, New York (2006) 